\title{
An ICT Architecture for Managed Charging of Electric Vehicles in Smart Grid Environments
}

\author{
S. Bohn, ${ }^{1}$ M. Agsten, ${ }^{2}$ O. Waldhorst, ${ }^{3}$ A. Mitschele-Thiel, ${ }^{1}$ \\ D. Westermann, ${ }^{1}$ and P. Bretschneider ${ }^{2}$ \\ ${ }^{1}$ Ilmenau University of Technology, 98693 Ilmenau, Germany \\ ${ }^{2}$ Advanced Systems Technology (AST) of Fraunhofer IOSB, 98693 Ilmenau, Germany \\ ${ }^{3}$ Institute of Telematics, Karlsruhe Institute of Technology (KIT), 76131 Karlsruhe, Germany
}

Correspondence should be addressed to S. Bohn; sven.bohn@iosb-ast.fraunhofer.de

Received 24 September 2012; Revised 27 December 2012; Accepted 10 January 2013

Academic Editor: Wenchuan Wu

Copyright $\odot 2013$ S. Bohn et al. This is an open access article distributed under the Creative Commons Attribution License, which permits unrestricted use, distribution, and reproduction in any medium, provided the original work is properly cited.

Growing shortage of fossil resources and an increasing demand of individual mobility worldwide require technology alternatives to existing mobility solutions. Electric vehicles (EVs) as one possible solution have moved into the focus of research and development. To maximize the positive environmental effect of EVs, it is proposed to charge them with respect to the availability of renewable energies. As the number of EVs will grow in the near future, their impact on the power distribution grid is no longer neglectable. Related research shows that unmanaged charging of EVs could result in overload situations or voltage instabilities. To overcome this, methods are proposed to manage the charging process holistically. Herein EVs become substantial elements of intelligent power grids (Smart Grids). As of today, research in the area of Smart Grids focuses mainly on either energy aspects or communication aspects while neglecting the interoperability of energy and communication related aspects. In this paper, an insight into Information and Communication Technology (ICT) aspects with respect to Managed Charging of EVs in Smart Grid environments will be given. Based on the use case of Managed Charging, requirements will be analyzed, results will be derived, and ICT solutions will be proposed with a set of recommendations for Smart Grid architectures.

\section{Introduction}

Due to the expected gain from approximately 1 billion existing vehicles worldwide as of today, up to 2.5 billion vehicles by $2030[1,2]$ will result in unknown supply issues, environmental issues, or even in shortages of fossil resources [3].

One possible solution to overcome this problem could be the global usage of electric vehicles (EVs) [1-3]. Their main advantage is the possibility for diversification of the transportation sectors primary energy sources. EVs have an environment-friendly image due to zero emissions while driving and potentially green charging by using renewable energies [3].

On the other hand, today's electricity generation is mainly centralized and based on fossil primary energy sources and nuclear power. The growing and politically driven deployments of renewable energies challenge the operation of power grids due to their volatile and decentralized infeed. In such decentralized environments, the power is generated in small units like photo voltaic (PV) systems, wind power systems, or combined heat and power (CHP) systems [4]. Storage applications and programs are required to harmonize volatile generation and demand (Demand Side Management (DSM) or Demand Response (DR)) $[3,4]$.

In such scenarios, EVs are highly interesting elements of DSM applications due to their relatively high charging power, significant energy demand, and ability to shift the load $[3,5,6]$. Related work shows that an increasing number of EVs will result in power grid overload situations and voltage stability issues if they are connected to the power grid in an unmanaged way $[3,7-9]$. One solution to overcome this would be the traditional network expansion by substituting transformers and power lines or adding primary equipment for reactive power compensation. Another more sophisticated approach would be the usage of Information and Communication Technology (ICT) in a Smart Grid environment $[3,8]$. 
Not only do Smart Grids imply decentralized generation and DSM programs, but also they represent a completely new paradigm for operation techniques for power distribution grids utilizing ICT [10-12]. Today there are many different definitions for a Smart Grid.

Differing even more so are the opinions about the components a Smart Grid infrastructure and a smart marketplace should be comprised off. It still remains an open question on how the different aspects of the Smart Grid can be supported by an ICT architecture.

The answer might be different for different use cases of the Smart Grid and what the Smart Grid should be. Considering the use case of Managed Charging of EVs as an example, the main question to be answered still is how an ICT architecture for the Smart Grid must be organized to coordinate charging of thousands of EVs in order to harmonize volatile and decentralized infeed (as a topic of the Smart Market dealing with Demand Response technologies dedicated for EVs) and to postpone traditional network expansion (as a topic of the Smart Grid dealing with power grid capacity).

In this paper, we present an ICT architecture for the Smart Grid that is tailored to the use case of Managed Charging of EVs. The architecture is organized as a hierarchy that reflects the structure of the power grid. Using the actual numbers of households from the power grid in Germany and the German federal state of Thuringia, we compute the workload on computing and communication devices in the ICT architecture and show that the architecture can efficiently support different deployment scenarios for both EVs and renewable energy sources in this area. The main results of our study are that a Smart Grid ICT architecture does not need to carry a high workload as stated in other publications $[11,13]$. Smart Grid communication architectures can be deployed by using small embedded systems if arranged in a hierarchical fashion which reflects the energy grid's hierarchy. This will enable the distribution grid to charge thousands or even millions of EVs in the future.

The remainder of this paper is structured as follows. Section 2 describes the distinction between Smart Market and Smart Grid. Section 3 explains the use case of Managed Charging. Section 4 derives ICT requirements for the use case and computes the workload on the architecture for different scenarios. Section 5 presents the hierarchical ITC architecture. Finally, concluding remarks are given.

\section{Smart Grid versus Smart Market}

In order to clearly differentiate Smart-Grid- and SmartMarket-related topics from each other, criteria were defined by the German Federal Network Agency (BNA). The main criterion for differentiating the two is the nature of the network capacity. The main criterion for differentiating the two is the network capacity, so to say the power or Watts, and the amounts of energy, so to say the energy or Watthours. In this case network capacity or power is related to the Smart Grid and energy is related to the Smart Market [4].
2.1. Smart Market. Smart Market clearly is related to the amounts of energy as a value that is generated at any kind of power plant, traded on the energy market, and consumed by any individual customer [4]. Energy producers and traders want to efficiently supply customers with affordable energy while respecting the constraints imposed onto them by the customers and government $[3,14,15]$. The energy market in Europe is going to be transformed to a market where every individual customer can choose its supplier due to his or her energy needs and comparable attributes on a liberalized market-a Smart Market rises [16, 17]. To interact with Smart Market instruments like Smart Meters and energy offers like TOU (TOU-Time of Use (Prices)), RTP (RTP-RealTime Pricing), or CPP (CPP_Critical Peak Pricing) [3, 14] requirements for the Smart Grid are needed. For this special purpose, an optimized and stable ICT infrastructure provides a base for dynamic Smart Market applications [18, 19]. In this context, dynamic means variable pricing conditions over different time periods.

2.2. Smart Grid. On the other hand, the grid has to be an enabler or server for Smart Markets. Not in the sense that a grid must be smart in order for a Smart Market to be established, but in the sense that a grid must be stable in every situation presented to it by the Smart Market [4]. Criteria for a stable operation are voltage stability, voltage quality, and utilization of assets like transformers, cables, overheat lines, and so forth. The traditional way of handling distribution grid problems is to expand the grid. However, changing transformers or cables can be quite expensive nowadays [3, $12,19]$.

A simple example to underline this aspect is the discussion about EVs and charging them at home. In a dedicated grid, no one can forecast when and where an EV will be charged [3]. Research shows [3, 7, 9, 20] that most EVs will be charged between $8 \mathrm{PM}$ and $6 \mathrm{AM}$ with a high charging power ranging from $3.6 \mathrm{kVA}$ to $44 \mathrm{kVA}$ within a 30 -minute to 6 -hour period. As a result the grid operator has to decide how to prepare the distribution grid effectively for the next 30 years. One way to prepare the grid is to calculate or forecast worst case scenarios with highly concentrated hot spots in place and time. Another way of doing it would be the assumption of a homogenous place and time distributed EV charging. Both ways have advantages and disadvantages, but both have one disadvantage in common. If the assumptions about time and places of charging EVs fail, the grid could become unstable or overstressed. The same problems occur if a huge amount of decentralized generation of renewable energy must be integrated into the distribution grid.

For example, based on forecasts, a grid expansion strategy was derived by the grid operator but the real situation occurred differently. What is the grid operator's strategy to get every possible situation covered now? Clearly he must expand the grid even more or completely considering all possible worst cases. This results in high costs and inefficient grid operation. On the other hand, a Smart Grid can include a dynamic grid capacity management which is supported by ICT. This approach will be discussed in this paper. 


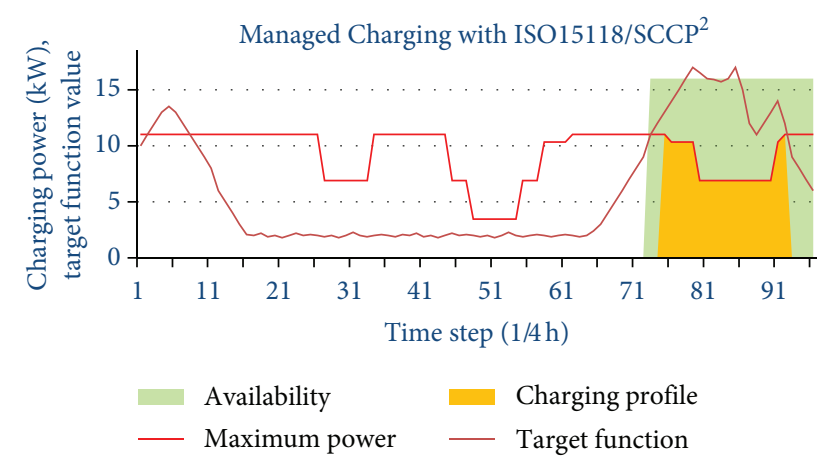

FIGURE 1: Managed Charging using target and restriction function.

As a summary, the Smart Grid and Smart Market focuses should not be discussed independently. Instead, a systems approach should be adopted in which Smart Grid technologies support Smart Markets and vice versa.

\section{Managed Charging of EVs}

A special use case is discussed in related research $[3,8$, $15,20]$. The question of how to integrate large amounts of EVs into the distribution grid deals with Smart-Market- and Smart-Grid-related topics. Green charging will be a major use case in Smart Market environments. In this case, EVs mainly charge when renewable energy is available. Among other motivations for green charging is the requirement for automotive OEMs to be sustainable in their future development. One reason is that the unmanaged charging process may not be physically coupled with the volatile renewable generation of electricity. Another strong focus will be priceoriented charging due to market conditions. Both focuses will be supported by the future development of a standardized charging interface between EVs and charging stations. The developments of ISO15118 CD2 [22] and Daimler/RWE Smart Charge and Communication Protocol [23] give insight.

In the use case of Managed Charging, a cost or target function over time indicates the best time slots for charging based on different profiles such as inexpensive charging, green charging, or any other profile. To avoid grid instabilities, a restriction function must be introduced. The role of the latter would be to enforce charging limits such as maximum charging power for every time slot [3]. Figure 1 visualizes the Managed Charging process using target and restriction functions.

3.1. The Managed Charging Process. In order to understand the need for the two functions (target and restriction function), the Managed Charging process will be described briefly. An extensive description can be found in $[3,8,21]$.

The process of Managed Charging can be described through a state machine. It starts when the EV is plugged in and ends when the EV is plugged off. After plugging in, the EV is waiting for the charging being turned on. Then the EV is charging until the battery of the EV is fully charged. The charging can be interrupted by the EV user at any time, and the process will finish. The state machine is depicted in Figure 2.

Mathematically there are two functions derivable. The availability av $(k T)$ of the EV while plugged in and the charge $\operatorname{ch}(k T)$, each depending on the time steps $k$ and the interval length $T$,

$$
\begin{gathered}
\operatorname{av}(k T)= \begin{cases}1 & \text { for } T_{\text {start }}^{\mathrm{av}} \leq k T \leq T_{\text {stop }}^{\mathrm{av}} \\
0 & \text { otherwise, }\end{cases} \\
\operatorname{ch}(k T)= \begin{cases}P & \text { for } T_{\text {start }}^{\mathrm{ch}} \leq k T \leq T_{\text {stop }}^{\mathrm{ch}} \\
0 & \text { otherwise, }\end{cases} \\
T_{\text {start }}^{\mathrm{ch}}=T_{\text {start }}^{\mathrm{av}} \\
T_{\text {stop }}^{\mathrm{ch}}=T_{\text {start }}^{\mathrm{ch}}+\frac{E(i)}{P(i, k T)} .
\end{gathered}
$$

$T_{\text {start }}^{\mathrm{av}}$ and $T_{\text {stop }}^{\mathrm{av}}$ mark the start and the end of a Managed Charging process, and $T_{\text {start }}^{\mathrm{ch}}$ and $T_{\text {stop }}^{\mathrm{ch}}$ mark the start and the end of the power drain. The interval length $T$ shall correspond to the 15-minute load profile which is used in the European grid. $E(i)$ is the energy demand of one EV. It is assumed to be stochastic and not unswayable. $P(i, k T)$ represents the battery charging process and is assumed to be based on the CCCV (constant current constant voltage) charging process which is used in modern battery systems.

For a (large) number $N$ of EVs, (1) and (2) expand to

$$
\begin{aligned}
\operatorname{Av}(k T) & =\sum_{i=1}^{N} \mathrm{av}_{i}(k T), \\
\mathrm{Ch}(k T) & =\sum_{i=1}^{N} \mathrm{ch}_{i}(k T) .
\end{aligned}
$$

These formulas represent a model that is able to describe the group behavior (availability and load) for a large number of EVs connected to the grid. Studies about this group behavior were done in $[3,8,21]$ and shall not be part of this paper.

Optimizing the charging process might be done for different applications and goals. The EV user might optimize for comfort and cost minimization. A DSO might optimize the process to optimize utilization of the equipment. A TSO has an interest in grid stability. In the studies of $[3,8,21]$, the interest was to optimize the charging process based on the generation of renewable energy from wind (Wind2Vehicle).

3.2. Target Function. The goal of the Managed Charging process is to shift the emerging charging processes from several EVs in a way that a maximum correlation with a given curve or function can be achieved. In the case of Wind2Vehicle $[3,8,21]$, the goal is to generate a load that is able to consume most/all the energy that is generated by wind.

Formula (2) will be extended by a shifting factor $\mathbf{s}$ :

$$
\operatorname{ch}(k T)= \begin{cases}P & \text { for } T_{\text {start }}^{\mathrm{ch}}+\mathbf{s} \leq k T \leq T_{\text {stop }}^{\mathrm{ch}}+\mathbf{s} \\ 0 & \text { otherwise. }\end{cases}
$$




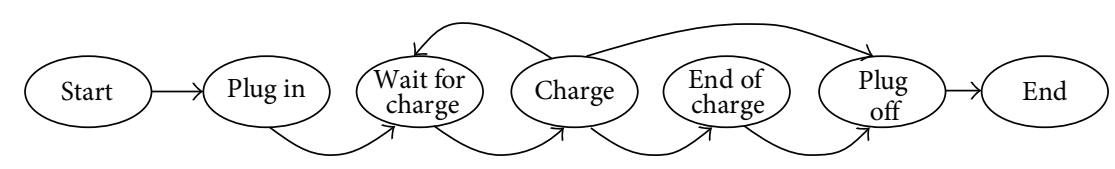

FIGURE 2: Managed Charging process.

The corresponding charge curve (6) can be extended to

$$
\mathrm{Ch}(k T, \mathbf{s})=\sum_{i=1}^{N} \mathrm{ch}_{i}(k T, \mathbf{s}(i)),
$$

while $\mathbf{s}$ represents the shifting vector of all charging processes.

In order to achieve a maximum correlation a target trajectory $C$ over the samples $k T$ has to be introduced. In the case of Wind2Vehicle, this is the total energy $E$ which is scaled up over the predicted power generation. In the case of solar energy, this could be the predicted generation by photovoltaics, or in the case of pricing, this could be the surplus energy available or predicted. It follows that

$$
E=\sum_{k=K_{B}}^{K_{E}} \sum_{i=1}^{N} \mathrm{ch}_{i}(k T, \mathbf{s}(i)),
$$

while $K_{B}$ describes the beginning and $K_{E}$ describes the end of the optimization horizon. The target trajectory follows as (in case of Wind2Vehicle)

$$
C(k T)=\frac{\operatorname{Wind}(k T)}{\sum_{k=K_{B}}^{K_{E}} \operatorname{Wind}(k T)} \times E .
$$

Based on the found formulas (8) and (10), the target function can be derived:

$$
\begin{gathered}
\min _{s}=\left(\|C-\mathrm{Ch}(\mathbf{s})\|_{2}^{2}+F(\mathbf{s})\right), \\
F\left(s_{i}\right)= \begin{cases}1 e 4 & \text { for } T_{\text {start }}^{\mathrm{av}} \leq k T \leq T_{\text {stop }}^{\mathrm{av}} \\
0 & \text { otherwise }\end{cases}
\end{gathered}
$$

The optimization problem is described as a nonlinear method of least squares over the samples $k T$ depending on the shifting factors s. In order to avoid shifting charging events outside the availability of the corresponding EV, invalid values will be penalized with $F\left(s_{i}\right)$. The optimization then is realized by a local search.

The target function does not have to be a single function following price or renewable generation. There could be several target functions from which the customer can choose. Alternatively, the customer could also generate a function which incorporates tradeoffs between several target functions.

3.3. Restriction Function. On the other hand, the restriction function is locally generated based on grid resource limitations at or up to the point of electricity consumption. One example here would be the maximum allowable current on the cables.
The maximum charging power of a connected vehicle is determined by the total utilization of the distribution grid including household service connections and other charging vehicles. In the event that a high target function value occurs the charging may still be limited to the actual capacity of the local distribution grid. A simple example would be the following. The load of the actual grid is limited to $100 \mathrm{kVA}$, but due to the actual high target function value, $10 \mathrm{EVs}$ are connected to charge at a rate of $20 \mathrm{kVA}$. This would overload the grid as there would be a total consumption of $200 \mathrm{kVA}$. Here the restriction function is used to limit the charging of each $\mathrm{EV}$ to $10 \mathrm{kVA}$ which would result in a total utilization of $100 \mathrm{kVA}$ which equals the maximum load [3].

This simple example shows the optimal utilization of the distribution grid as a tradeoff between the target function and the restriction function. Additional functions may be introduced in order to optimize Managed Charging of electrical vehicles even further or to customize the end users choice of charging premises. In our understanding, a similar method is necessary for charging stationary reservoirs like battery packs or pump storage plants.

The calculation scheme for the restriction function with special focus on avoiding violating the voltage band as per EN50160 [24] and for the utilization of the distribution grid is shown in Figure 3.

The described approach requires the integration of ICT in the distribution grid. Each consumer's load must be monitored at any time period and forecasted for the period an EV is charging. To transport necessary information and manage the charging process over the whole distribution grid, an architecture which fulfills the communication requirements as shown in Figure 4 is needed.

\section{ICT in the Smart Grid}

At this point it should be understood that the two functions, target and restriction, are needed to utilize the grid in an optimal way and implement the Managed Charging process. But where do these functions come from? What data is needed? The traditional way of network expansion is not supporting this approach. What is needed is a Smart Grid that is able to communicate information about actual conditions on the grid and to react on certain or critical circumstances in order to keep the grid in a safe operation range.

4.1. Smart Meter. Many companies call their metering products Smart Meters, starting from simple electronic meters that have an LC display on it or meters that are able to transmit their actual reading via ZigBee or PLC. These meters are not considered Smart Meters in this context or devices suitable for a Smart Grid at all-they are dumb 

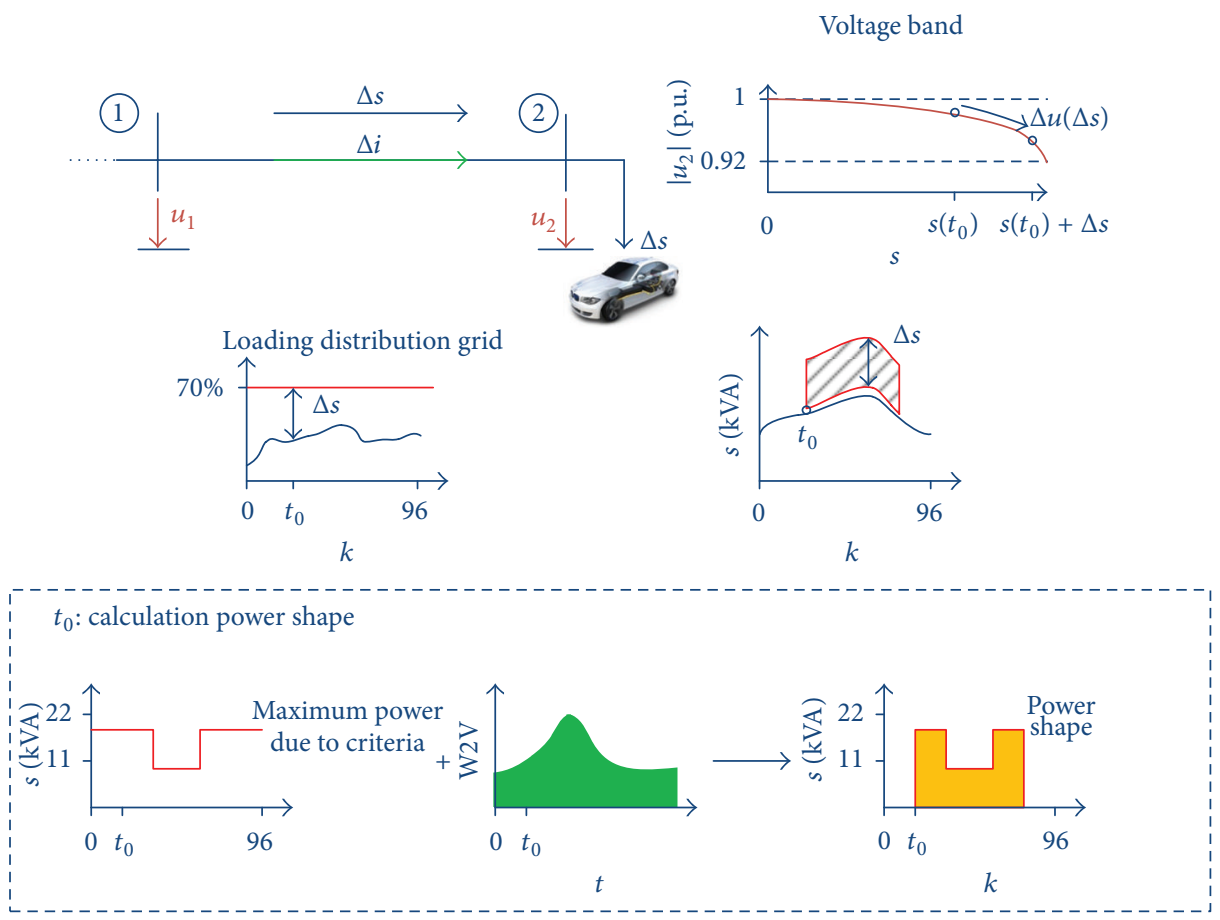

FIGURE 3: Calculation of the restriction function [3, 21].

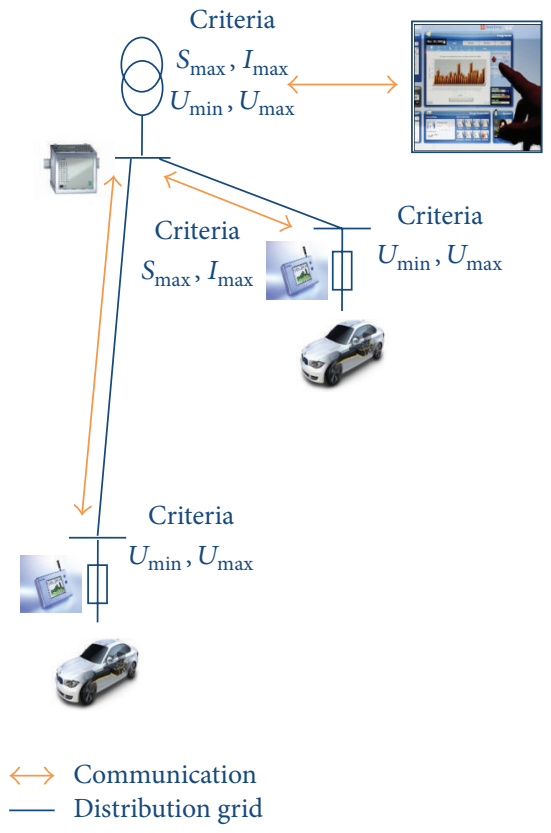

Figure 4: Architecture [21].

intelligent devices. More advanced products that are able to communicate additional information about the actual service connection or connection point of the grids that have a bidirectional communication interface are considered Smart Meters in this context. These Smart Meters interact with the grid, the customer, and the market. They will enable all participants to manage power supply. The Smart Meter becomes an active element and will be a sensor as well as an actor.

As mentioned earlier, the minimum additional information needed consists of at least voltage per phase, current per phase, phase angle per phase, timestamp of the actual measurement, and meter ID in order to identify the measurements. These minimum requirements are needed in order to determine the actual state of the grid at this specific service connection or connection point. Aggregating the information of all Smart Meters that are ideally installed at each home service connection, charging point, or infeed point allows the generation of a time-dependent overall picture of the actual status of the distribution grid. Related work in this field is done by Siemens with their concept "Augen im Netz" (Power Snapshot Analysis) [19].

In this paper, we further expand the definition. A Smart Meter should be able to do even more than that mentioned above. A Smart Meter should not be viewed as a device in the traditional sense of a meter anymore. It should be a function among others in a device. The traditional metering function as of today should be a calibrated and certified function residing inside a device that implements the Smart Meter. In addition to that the Smart Meter should be some kind of smart device like today's smart phones that are able to load additional functions or features. Comparable as in the smart phone market, it should be able to load additional Apps in order to add additional functions or features as depicted in Figure 5. 


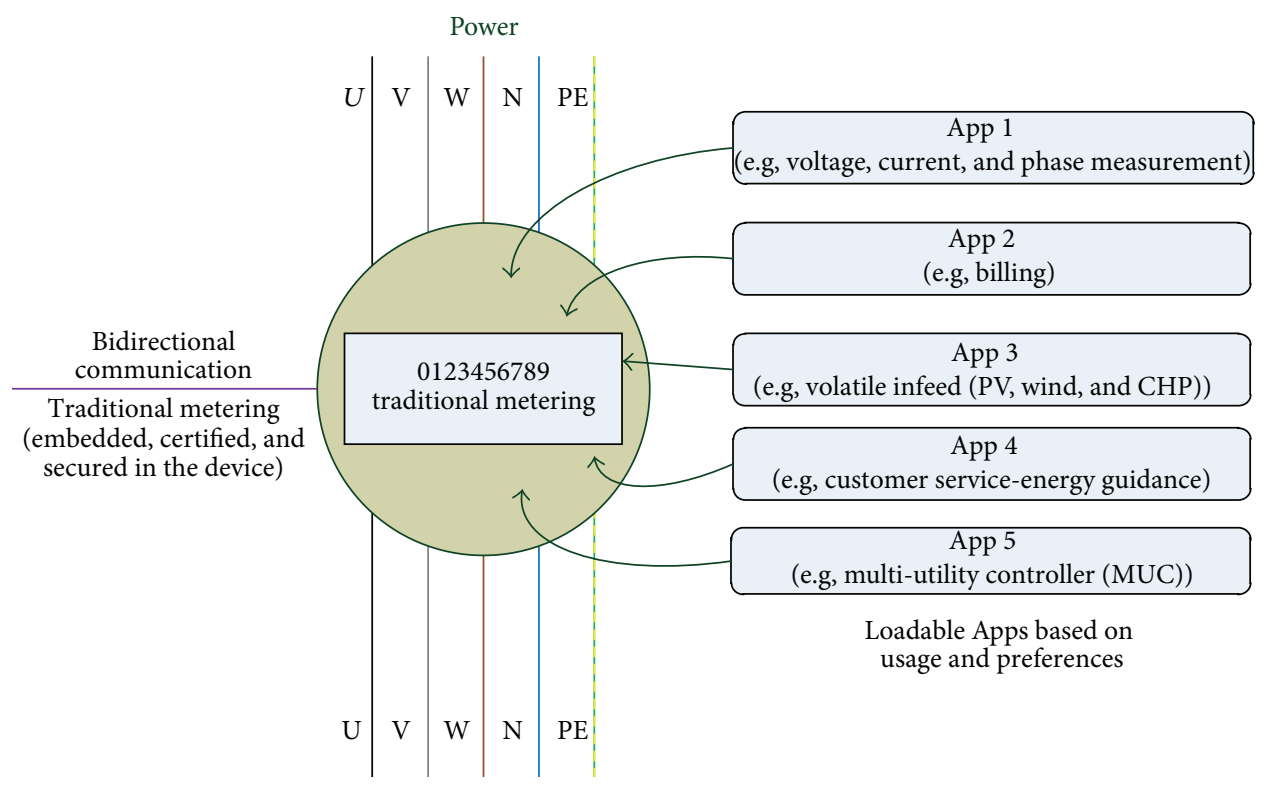

Figure 5: Smart Meter.

Whereby the features are not limited to the usage of the grid operator. Features should be usable by different users such as the grid operator, the energy provider, or the end user. These features may also include aspects of the grid like grid stability, billing, automation, infeed, and so forth.

To summarize, a Smart Meter has to be more than an energy accumulation device. It needs to provide additional services and needs to communicate additional information to the different players of Smart Grid, Smart Market, homeowners, and EV users in order to establish a Smart Grid and add value to the society.

4.2. Estimation of Data Volume Generated by Smart Meters. Based on the assumption that an Advanced Metering Infrastructure (AMI) is established using Smart Meters as described above, the question is what are the requirements for a communication infrastructure? Comparing the advantages and disadvantages of different approaches, it is necessary to evaluate the data volume or workload in each scenario.

There are nearly 40 Million households in Germany as of today. This number will increase to 41 Million household by 2020 and will stay stable after that [25]. There are nearly 100 different measurements or information that a Smart Meter is able to communicate [18] as of today. Furthermore, the amount of information will grow in the future as new applications will be discovered and additional information is needed.

At an average of 26 bits per measurement, a row data volume of 325 bytes is generated for each meter reading at every single Smart Meter and needs to be communicated over a communication infrastructure of some kind. Assuming an Internet Protocol (IP) based communication is used to communicate these measurements, an overhead of at least 54 bytes (IPv4/UDP) is added to the measurements generating a data volume of 379 bytes per meter reading to be transmitted on the communication infrastructure. Similar results are expected using other wired communication technologies like PLC or wireless technologies like WiMAX or LTE. The last two mentioned technologies build on IP as well.

Ignoring packet loss and retransmissions, there will be a data volume of approximately 15 GB for every meter reading interval in total for Germany. Herein the number of Smart Meters installed at companies (3.6 Million in 2009 [26]) and energy generation plants is not included. Also the bidirectional communication for controlling DSM, LLM, and so on is not included in this calculation.

In order to calculate the total amount of generated data, it needs to be understood how the grid operates. As of today, there is a 15-minute load profile to forecast the power consumption of the next 15 minutes. In order to implement ICT support for this already established method, it is sufficient to collect one meter reading at each 15-minute interval. Therefore, presented data volumes in this paper are based on this 15-minute interval. The 15-minute load profile is depicted in Figure 6 as a dotted line.

Improving grid control requires shorter meter reading cycles because the actual load does not follow the discretized load profile. This is shown in Figure 6 where the solid line represents the actual load. In this case, meter reading cycles should be increased to once per minute. However, in this paper, the focus lies on the 15-minute load profile.

\section{Proposed Communication Infrastructure}

5.1. Centralized Approach. Assuming a centralized approach, is used there will be approximately $13 \mathrm{~GB}$ of raw data that will need to be communicated, processed, and stored every 15 minutes. This amount of data cannot be transmitted all at 


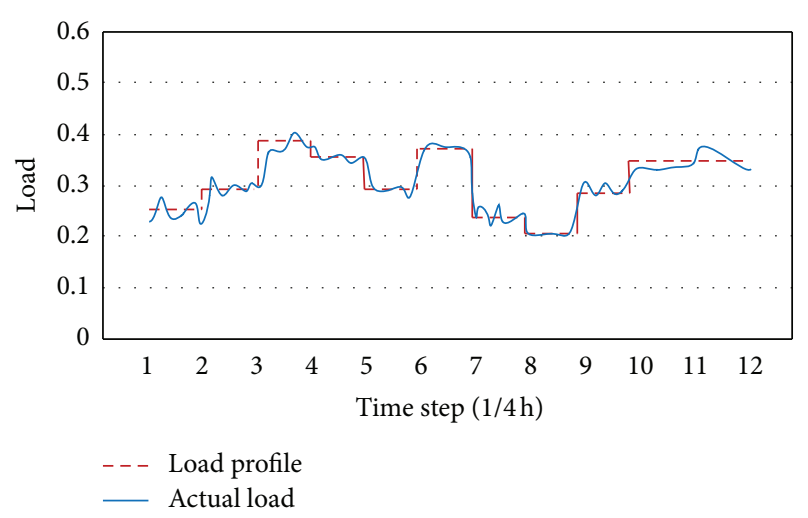

FIgURE 6: Load profile versus actual load.

once in a short time. Time inconsistency, loss of information, and retransmissions will occur. Also all or at least some of the data need to be stored for further processing, logging, or billing purposes. In the event that all data is stored, $52 \mathrm{~GB}$ will accumulate every hour which will amount to $1.2 \mathrm{~TB}$ per day. Storing data for one year, the billing interval in Germany will produce $455 \mathrm{~TB}$ of data. However, it is not necessary to store all the data and the data which must be stored may be compressed.

Even if these numbers show an implementable solution using today's technology, the centralized approach appears slow, instable, unsuitable, and inefficient. Peering into the future, increases in data volume and frequency of meter readings would quickly overwhelm the centralized approach. Taking meter readings every minute would produce $13 \mathrm{~GB}$ of raw data per minute, $780 \mathrm{~GB}$ every hour, 18.7 TB every day, and 6832.8 TB per year, for German households only.

5.2. Decentralized Approach. The decentralized approach is a solution where several instances are spread all over Germany to handle the amounts of data collected from Smart Meters. This could be some kind of cloud approach. It might be more feasible than the centralized approach but still appears inefficient. The decentralized approach can handle the same amount of data much quicker and can respond to changes in the grid operation in a much shorter time. However, it will generate additional traffic over the communication infrastructure due to synchronization between the computing instances.

5.3. The Hierarchical Approach. When designing a Smart Grid, one should employ an architecture which reflects the hierarchical nature of the grid. Therefore, the grid or its requirements should define the base of the structure of the communication system and not the other way around because goal of the Smart Grid is to control energy supply using ICT.

Figure 7 presents the hierarchy of today's energy grid. Clearly, several layers divided by a transformer can be seen. Each layer or voltage level has its own unique physical characteristics and requirements. As a result, each layer is a separate entity which interfaces with other layers through a transformer. A more technical visualization of the grid is given by Figure 8 .

For automation and control purposes, each entity can be dealt with as a closed system. For the Smart Grid, this means that not all information collected at a Smart Meter must be held available in a common way as implemented in the centralized or decentralized approach. There is no need to create some kind of big picture of the overall grid. Clearly, it can be broken down into several closed systems that report only aggregated information to higher level loops in a hierarchical fashion.

Known from the area of home automation is the principle of utilizing multiple sensors spread throughout the home to collect data and send it to a central instance which performs all the processing. All system data are used for control and automation, but only a few aggregated data are communicated to the outside world or user. Therefore, home automation could be understood as one layer of this hierarchical approach and can even be integrated into the Smart Grid hierarchy as level 8 or level 9 with respect to Figure 7.

Starting from the edge of the grid (level 7), this means that each substation providing electricity for its household service connections will be equipped with some kind of data concentrator. This data concentrator collects data from each connected Smart Meter. In order to optimize the utilization of this last mile, information only from this last mile is needed. Information from other Smart Meters not connected to this last mile is irrelevant.

On the other hand, each substation acts like a service connection to the level above. Therefore, the data concentrator of each substation needs to act as a Smart Meter as well. It measures the actual situation like a Smart Meter would do, and it uses aggregated information from its connected Smart Meters and reports the actual conditions to the level above. As a result, the higher level data concentrator only collects data from its substations and does not need information from each and every Smart Meter. Figure 9 incorporates the hierarchical ICT approach into the grid depicted in Figure 8.

Following this approach will drastically reduce the amount of data transmitted over the Smart Grid communication infrastructure and will only use data where they are needed.

This approach will also support the effort in providing a secure and private communication across the Smart Grid because only a few data or even aggregated and therefore anonymous data will be seen at the point of intrusion.

Nevertheless, some data need to be communicated beyond the actual data concentrator. This information could be faults, billing information, some kind of nongrid data, and so forth. In this case, the functionality of a gateway should be introduced to the Smart Grid. The gateway functionality can be implemented in the data concentrator or any other Smart Grid device. This gateway is able to transport selected information beyond the actual data concentrator or into a non-Smart Grid communication infrastructure like the Internet.

Figure 9 visualizes the proposed hierarchical approach for the communication infrastructure. The green marked nodes 


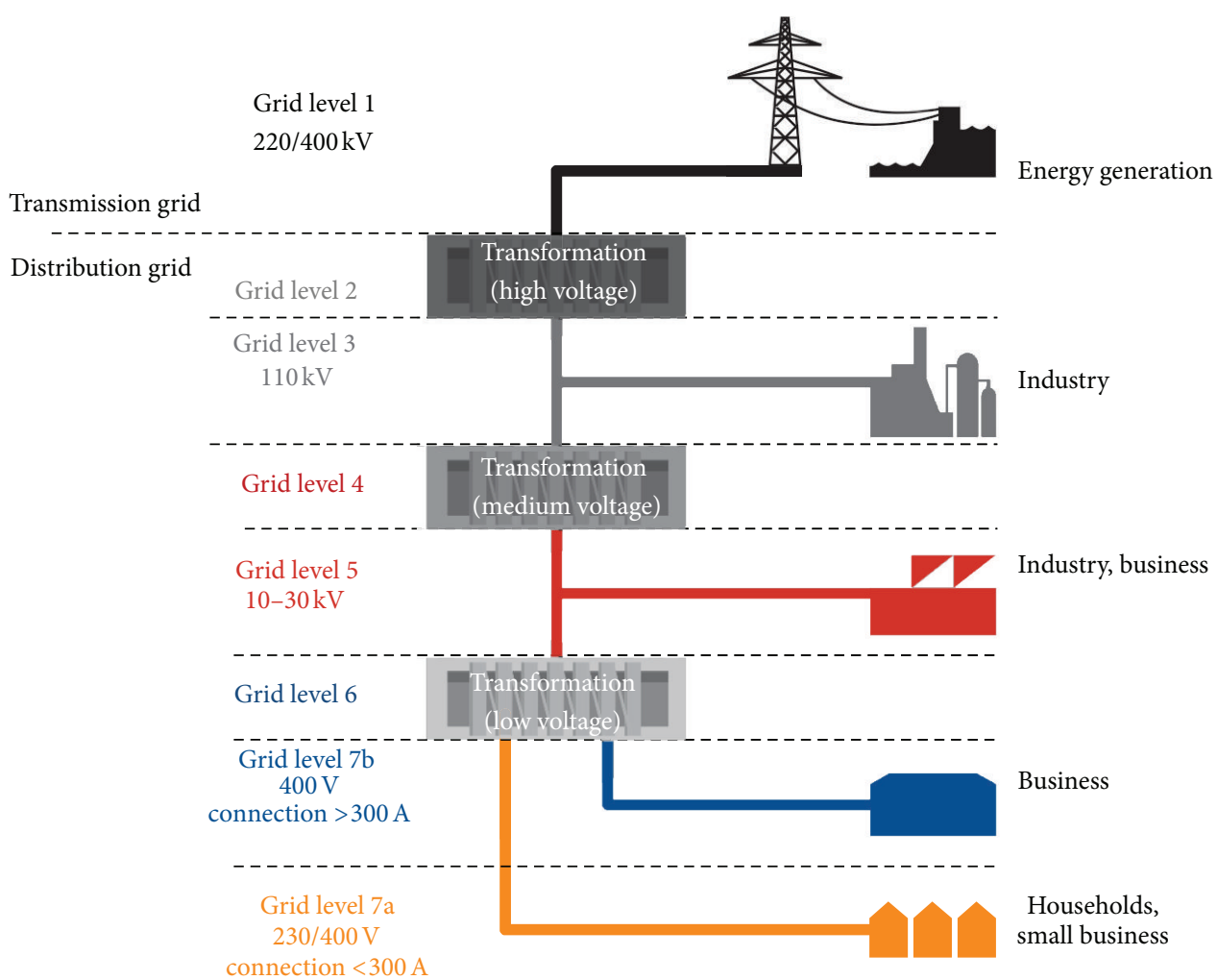

FIGURE 7: Hierarchy of the energy grid (source: http://www.iwb.ch/media/Strom/Dokumente/iwb_factsheet_netzebenen_0309.pdf).

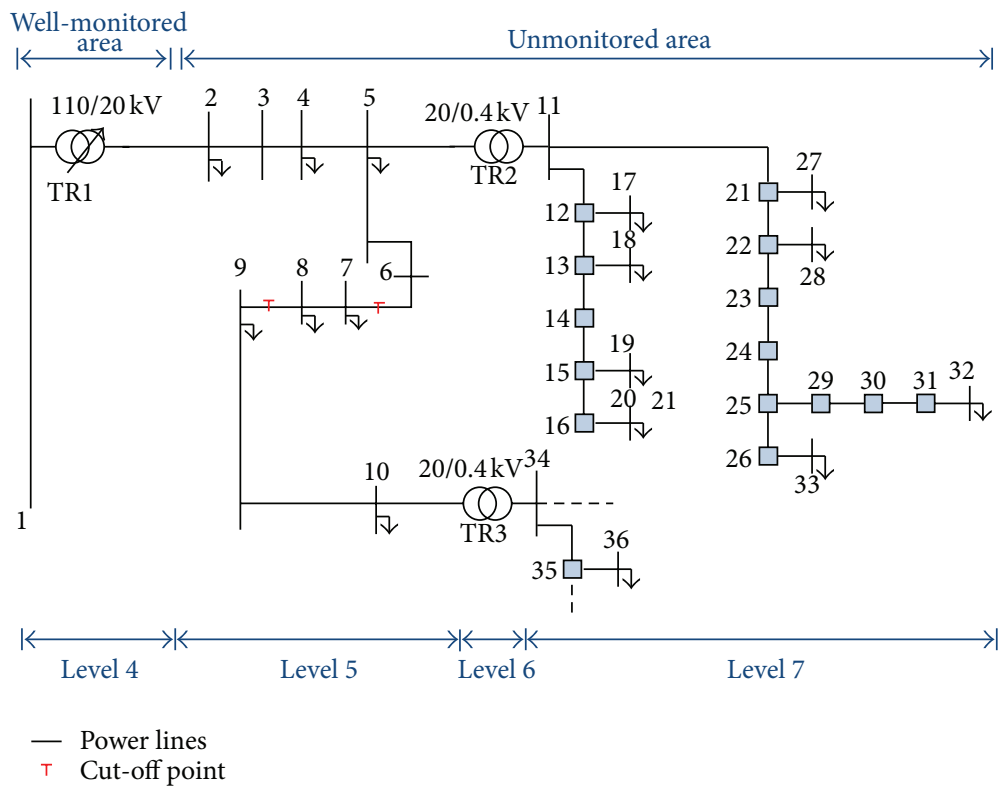

FIGURE 8: Reference grid.

are Smart Meters. They also might be data concentrators or gateways for home automation as mentioned before. The nodes marked red are data concentrators for level 7 collecting information from its associated Smart Meters. They are also gateways to the level 5 communication infrastructure or a nongrid communication infrastructure. So, each red node could have a connection to another communication infrastructure. On the level 5 side, these nodes are Smart Meters providing information about the grid at their specific connection, at the actual substation.

This hierarchical structure continues through all layers up to the highest. As of today, the high voltage transmission 


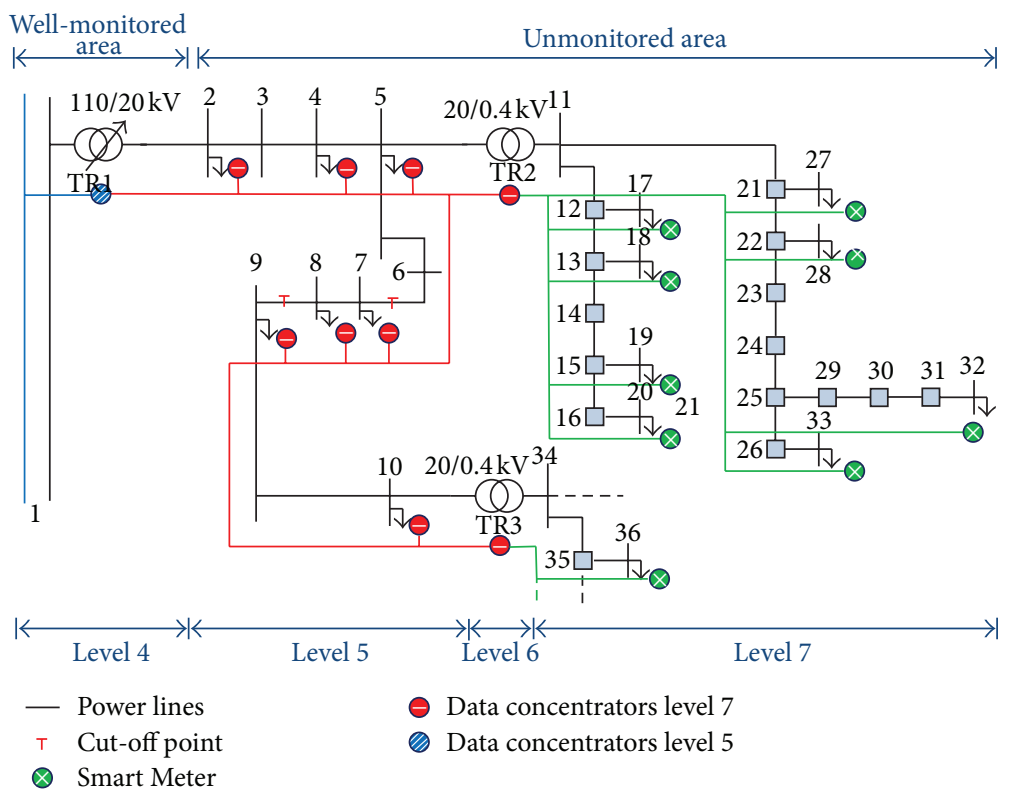

Figure 9: Hierarchical approach.

level is well monitored and automated. However, additional information from lower layers will further support control of the high voltage layer.

Based on kindly provided data from a regional grid operator, the reduction of the communication effort should be calculated. In the state of Thuringia, there are 87 high voltage transformer stations (level 4), approximately 9500 substations (level 6), and approximately 400,000 home service connections (level 7) [12]. A central approach would lead to approximately $130 \mathrm{MB}$ of raw data for every meter reading cycle. In opposite to this, the proposed hierarchical approach produces approximately $14 \mathrm{~KB}$ of data every reading cycle at an average of 42 Smart Meters per substation. This small amount of data can be communicated through slow communication systems like narrow-band PLC, even if the meter reading rate goes up to one per minute. For the level 5 communication, there are 9500 substations (level 6) that communicate to 87 high voltage transformer stations (level 4) and generate on average approximately $35 \mathrm{~KB}$ of raw data.

Given this small workload, small embedded devices can be used as data concentrators and Smart Meters. No big systems like server farms or optical fiber communication are needed to handle the amount of data. A Smart Grid can be implemented with small and inexpensive embedded devices.

5.4. Managed Charging. Coming back to the use case of Managed Charging, each charging point, either public or at home, has to have a Smart Meter implemented and needs to act as a gateway in order to communicate to the vehicle. Only communication to the vehicle's charger allows automating the charging process using the target and restriction functions.

The target function might be altered by the level 6 data concentrator. The restriction function is generated by the level 6 data concentrator based on the actual status of the grid that was measured by related Smart Meters and grid limitations that are provided by the grid operator. Limitations can be provided statically by programming the limitations into the data concentrator or dynamically by communicating the limitations to the specific data concentrator. Here algorithms for LLM are used to generate the restriction function [3]. Then, the restriction and target functions are communicated to the charging points and vehicles. In order to implement closed loop control, the charging process must be monitored like the rest of the grid to adjust the restriction function over time, to react to critical situations, and to optimize the grid utilization. Figure 10 shows the charging scenario on the grid.

Optimization algorithms may run within the data concentrator in order to compute an optimal utilization of the grid (Figure 11) to fulfill the needs of each consuming household and charging vehicle with respect to the generation of renewable energy.

In case there is a permanently higher demand for power than that the local limitations are able to provide, in Germany that means usually above $70 \%$, then traditional network expansion will be required. However, using ICT enables the grid operator to acquire information about the distribution grid and utilize it in a maximum way before replacing cables and transformers to expand the grid. In our view, this will be the most efficient and cost effective way to serve energy requests and demands. 

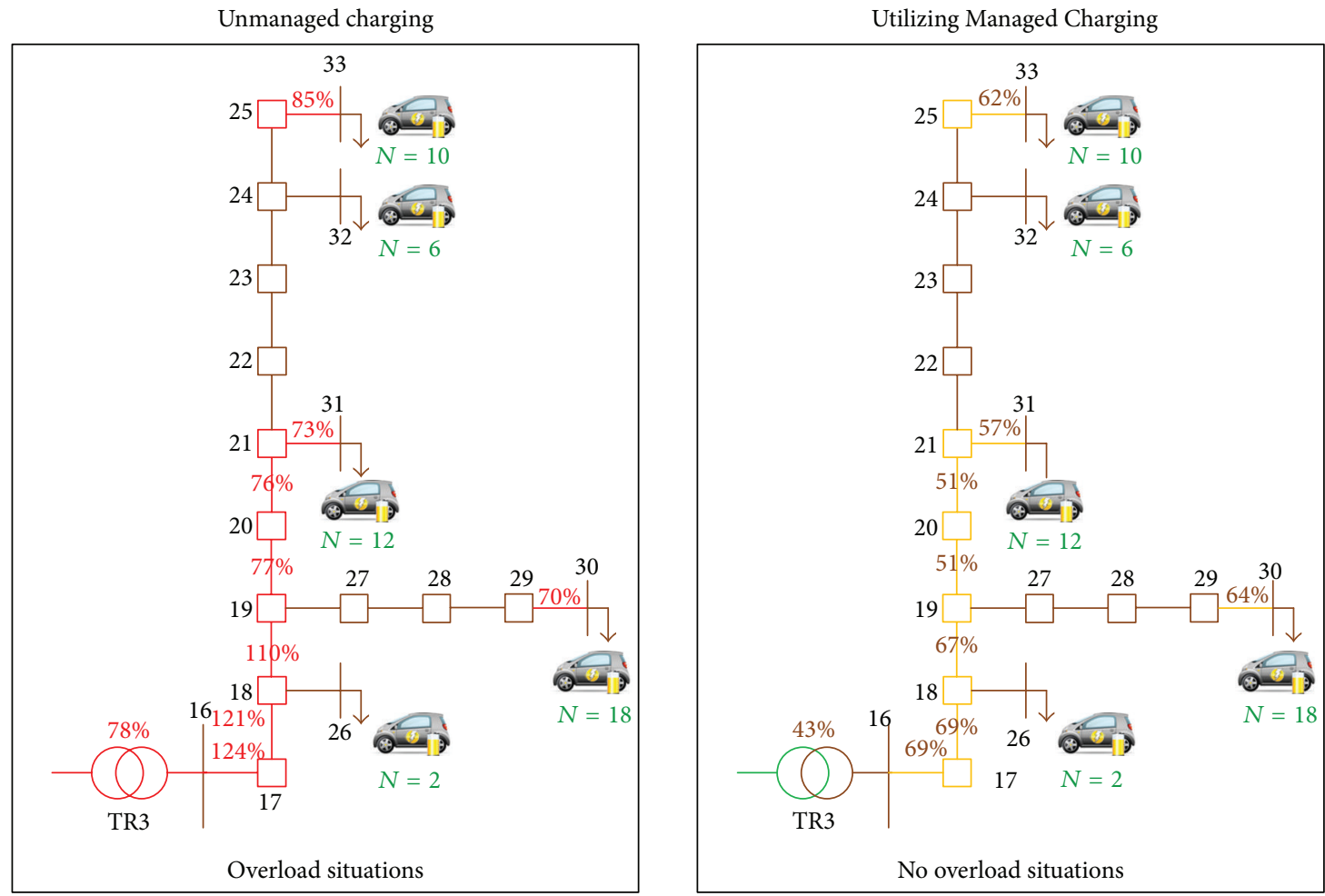

FIGURE 10: Charging scenario (overload situations).

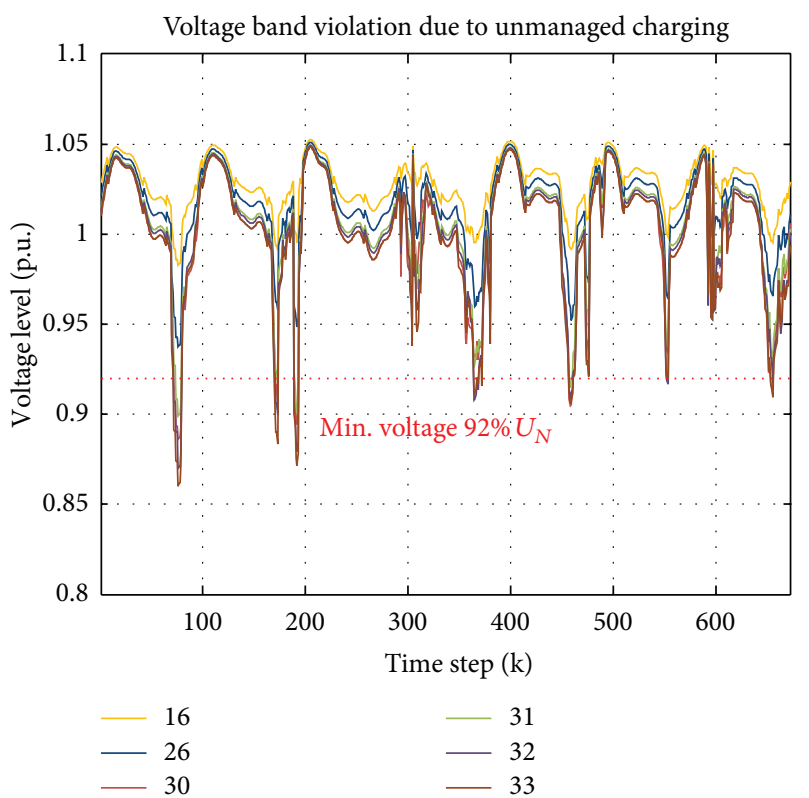

(a)

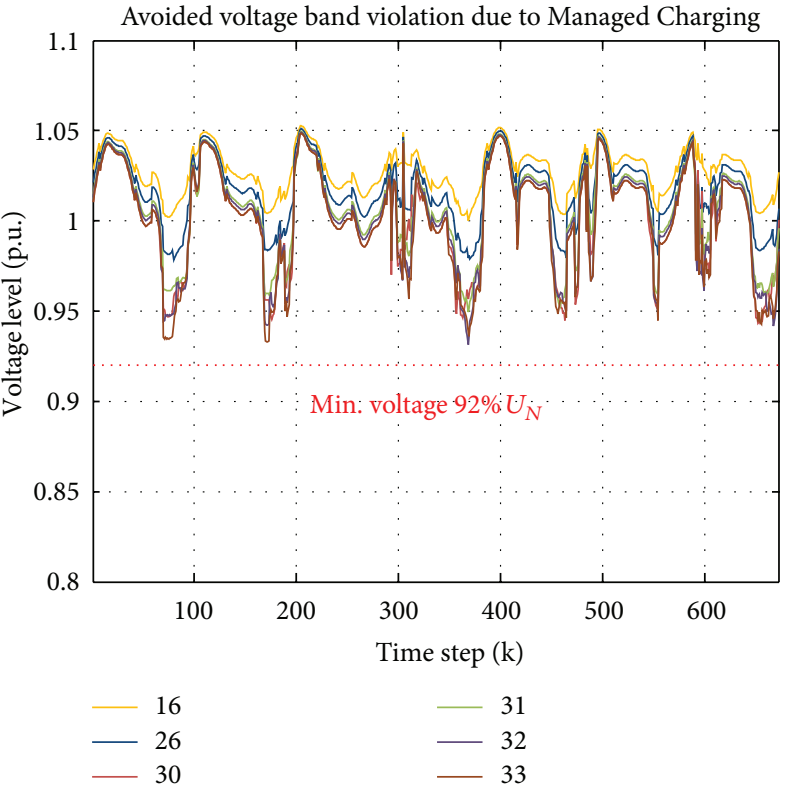

(b)

FIGURE 11: Charging scenario (violation of the voltage band).

\section{Conclusion}

The Smart Grid is expected to affect all levels of the grid including generation, transmission, and distribution. Therefore, new ICT architectures tailored to meet the grids' requirements are needed to handle the future supply and demand of volatile energy.

In this paper, it was shown that only a few tens to hundreds of Kbytes of data need to be communicated when using a hierarchical infrastructure. Therefore, a Smart Grid ICT 
architecture can be established using small and inexpensive embedded devices similar to DSL boxes or WiFi routers.

\section{References}

[1] J. Dargay, D. Gately, and M. Sommer, "Vehicle ownership and income growth, worldwide: 1960-2030," Energy Journal, vol. 28, no. 4, pp. 143-170, 2007.

[2] D. Sperling and D. Gordon, Two Billion Cars: Driving Toward Sustainability, Oxford University Press, 2009.

[3] M. Agsten, Einfluss gesteuerten Ladens von Elektrofahrzeugen auf die Netzbetriebsführung bei volatiler Windeinspeisung [Ph.D. dissertation], Ilmenau University of Technology, 2011.

[4] Bundesnetzagentur, “Smart Grid' und "Smart Market”-eckpunktepapier der Bundesnetzagentur zu den Aspekten des sich verändernden Energieversorgungssystems," December 2011.

[5] M. Agsten, S. Schlegel, and D. Westermann, "Load management potential of electric vehicles," Automatisierungstechnik, vol. 59, no. 3, pp. 188-195, 2011.

[6] M. Agsten, S. Schlegel, D. Westermann, and M. Ifland, "Utilizing battery electric and plug-in hybrids," in IEEE General Meeting, Detroit, Mich, USA, 2011.

[7] C. Rehtanz and J. Rolink, "Conditions for the demand side management of PHEVs and EVs," in ETG Kongress, Düsseldorf, Germany, 2009.

[8] M. Agsten, S. Schlegel, and D. Westermann, "Impact of managed charging of EVs on distribution grids with volatile wind generation," Automatisierungstechnik, vol. 60, no. 2, pp. 92-101, 2012.

[9] P. Bauer, Y. Zhou, J. Doppler, and N. Stembridge, "Charging of electric vehicles and impact on the grid," in Proceedings of the 13th International Symposium on Mechatronics (MECHATRONIKA '10), pp. 121-127, Teplice, Slovakia, June 2010.

[10] M. Erol-Kantarci and H. T. Mouftah, "The impact of smart grid residential energy management schemes on the carbon footprint of the household electricity consumption," in Proceedings of the 4th IEEE Electrical Power and Energy Conference (EPEC '10), Halifax, Canada, August 2010.

[11] A. Aggarwal, S. Kunta, and P. K. Verma, "A proposed communications infrastructure for the smart grid," in Proceedings of the Innovative Smart Grid Technologies Conference (ISGT '10), pp. 1-5, January 2010.

[12] T. Roscher, "Vorstellung und aktivitäten unseres unternehmens im kontext smart grid," in Proceedings of the 2nd Workshop on Smart Grids, Ilmenau, Germany, 2012.

[13] V. K. Sood, D. Fischer, J. M. Eklund, and T. Brown, "Developing a communication infrastructure for the smart grid," in Proceedings of the IEEE Electrical Power and Energy Conference (EPEC '09), October 2009.

[14] M. Klobasa, Dynamische Simulation Eines Lastmanagements und Integration von Windenergie in ein Elektrizitätsnetz auf Ladesebene Unter Regelungstechnischen und Kostengesichtspunkten, Zürich, Switzerland, 2007.

[15] BMW Group AG, Abschlussbericht zum Forschungsvorhaben KlImaentlastung Durch den Einsatz Erneuerbarer Energien im Zusammenwirken mit Emissionsfreien ElektrofahrzeugenMINI E 1.0, München, Germany, 2011.

[16] Europäische Kommission, "European Technology Platform for the Electricity Networks of the Future," 2010, http://www .smartgrids.eu/.
[17] Europäische Parlament und Europäischer Rat, "Richtlinie 2006/32/EG des europäischen Parlaments und des Rates über Endenergieeffizienz und Energiedienstleistungen und zur Aufhebung der Richtlinie 93/76," 2006.

[18] Siemens, "AMIS Formate Zähler und DB," unpublished.

[19] A. Abart, M. Stifter, B. Bletterie et al., "Eyes to the grid': new ways to analyze electrical low voltage networks," Elektrotechnik und Informationstechnik, vol. 128, no. 4, pp. 122-127, 2011.

[20] Vattenfall Europe Innovation GmbH, BMW Group AG, TU Berlin, TU Chemnitz, TU Ilmenau, "Steigerung der Effektivität und Effizienz der Applikationen Wind-to-Vehicle (W2V) sowie Vehicle-to-Grid (V2G) inklusive Ladeinfrastruktur," Hamburg, Germany, 2011.

[21] M. Agsten and D. Westermann, "Verfahren zur gesteuerten Entnahme elektrischer Energie aus einem Verteilernetz für elektrische Kraftfahrzeuge (pending)," Germany Patent DE 10 2011104 903.0, June 2011.

[22] International Organization for Standardization, "ISO 15118 CD2 Road vehicles, Communication protocol between electric vehicle and grid," 2011.

[23] R. W. E. Daimler, "Communication Protocol between Electric Vehicles and Charging Units," 2009.

[24] E. N. 50160:2010, "Voltage characteristics of electricity supplied by public distribution networks," 2010.

[25] Federal Statistical Office (Destatis), "Entwicklung der Privathaushalte bis 2030-ergebnisse der Haushaltsvorausberechnung," March 2011.

[26] Federal Statistical Office (Destatis), "Unternehmen und Betriebe im Unternehmensregister," https://www.destatis.de/ DE/ZahlenFakten/GesamtwirtschaftUmwelt/UnternehmenHandwerk/Unternehmensregister/Aktuell.html?nn=50674. 

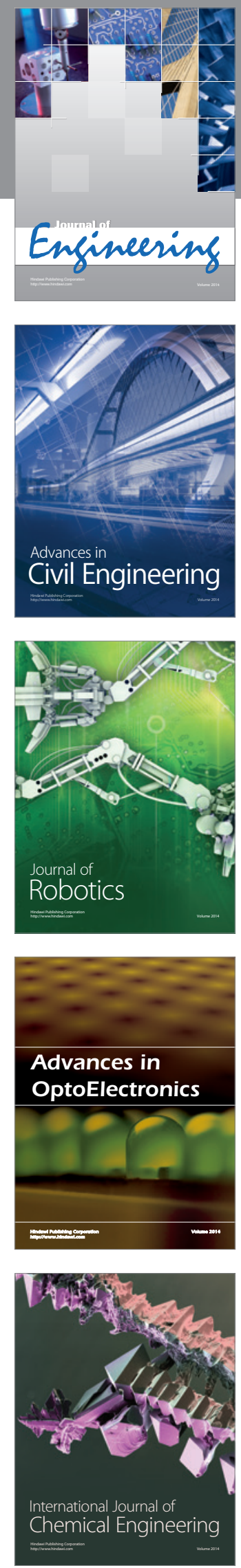

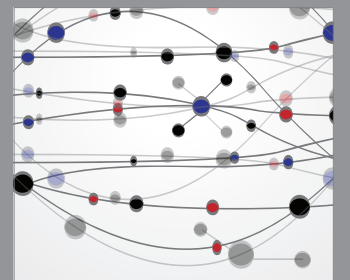

The Scientific World Journal
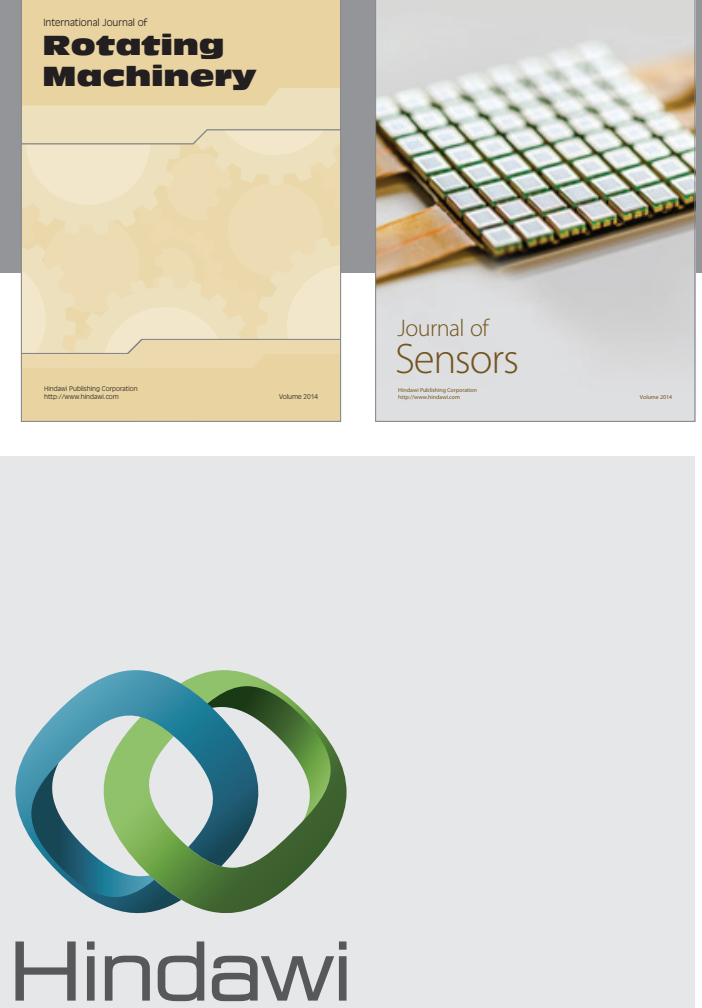

Submit your manuscripts at http://www.hindawi.com
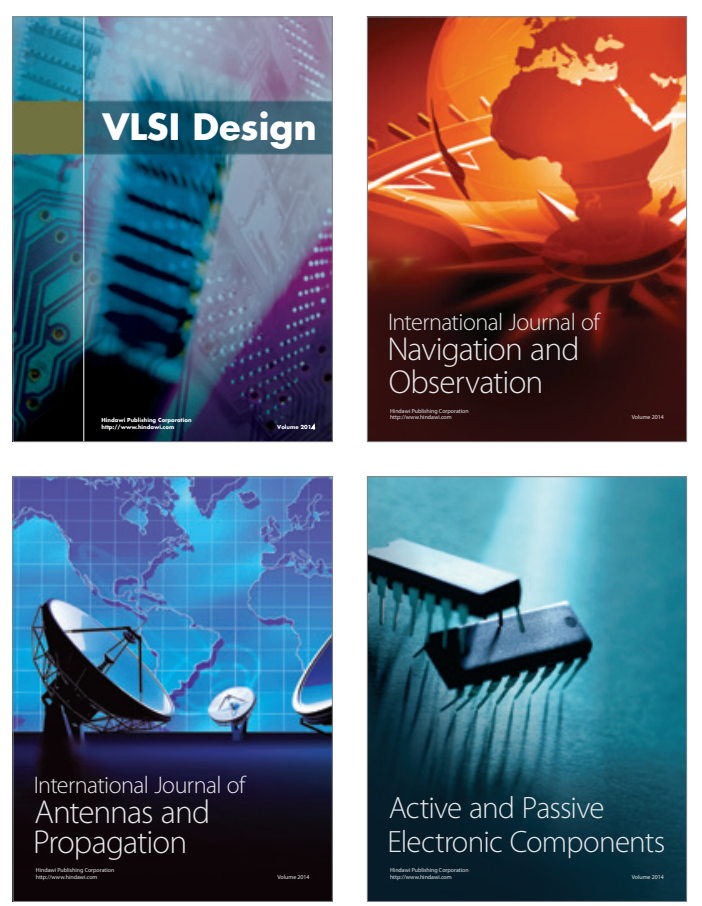
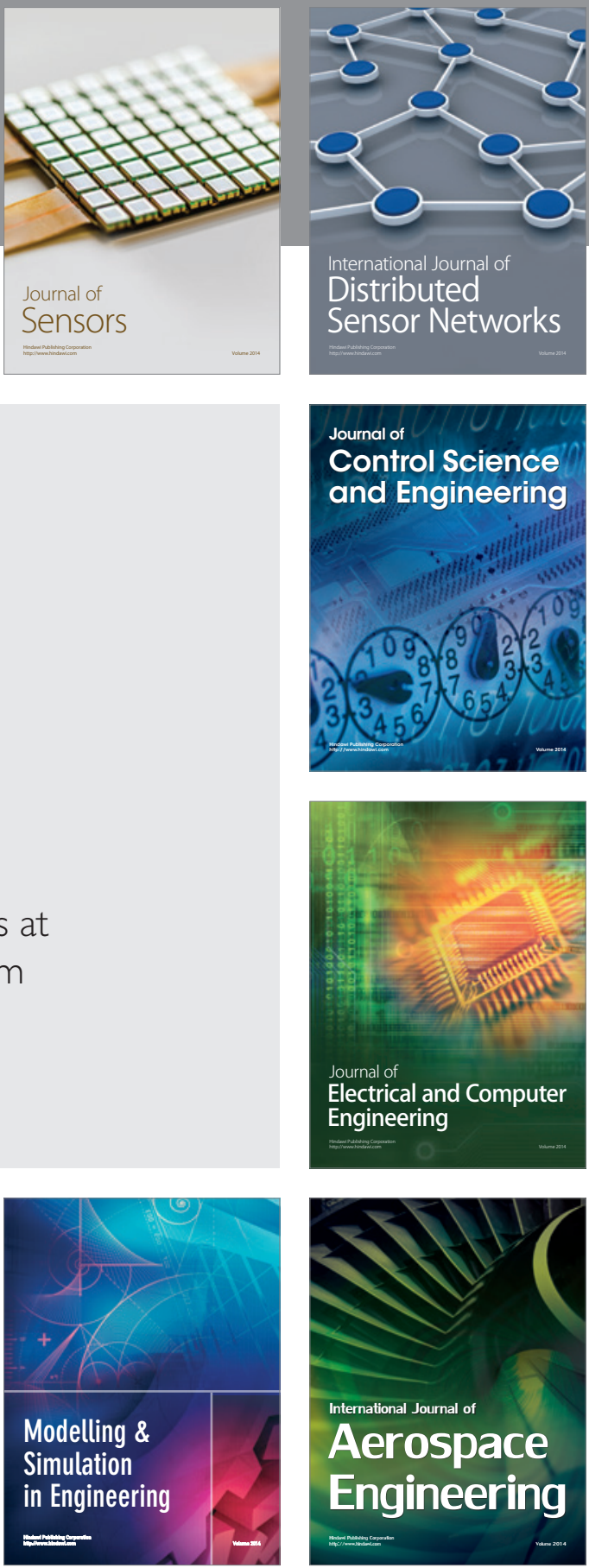

Journal of

Control Science

and Engineering
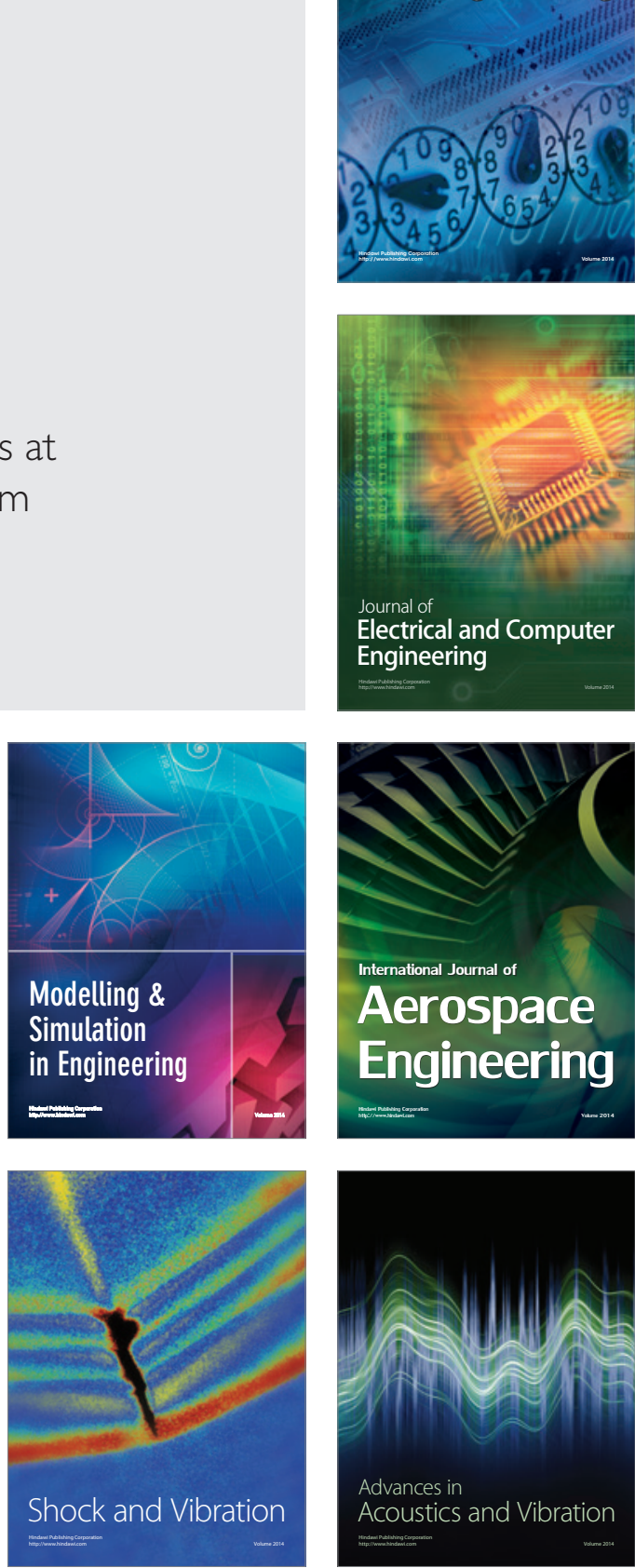